\title{
On Polyharmonic Riemannian Manifolds
}

\author{
R. Schmmmg and J. Kowolik
}

Eine natürliche-Verallgemeinerung der harmonischen Mannigfaltigkeiten wird behandelt: eine Riemannsche Mannigfaltigkeit heißt $k$-harmonisch oder polyharmonisch, falls sie eine nur vom geodätischen Abstand $r=r(x, \dot{y})$ bzw. von der Syngeschen Funktion $\sigma=\sigma(x, y)$ abhängige nicht-konstante $k$-harmonische Funktion - d. h. eine Lösung $F$ von $\Delta^{k} F(\sigma)=0$ - gestattet. Bestimmte Theoreme verallgemeinern sich von harmonischen auf polyharmonische Mannigfaltigkeiten.

Рассматривается естейственное обобщение гармонических многообразий: риманово многообразие называется, $k$-гармоническим или полигармоническим, если оно позволяет непостоянную $k$-гармоническую функцию, зависящая только от геодеаического расстояnил $r=r(x ; y)$ или от функции Синга $\sigma=\sigma(x, y)$, значит - решение уравнения $\Delta^{k} F^{\prime}(\sigma)=0$. Некоторые теоремы о гармонических многообразиях обобщаются на полигармонические многообразия.

A natural generalization of the harmonic manifolds is considered: a Riemannian manifold is called $k$-harmonic or polyharmonic if it admits a non-constant $k$-harmonic function depending only on the geodesic distance $r=r(x, y)$ or rather on Synge's function $\sigma=\sigma(x, y)$, i.e. a solution $F^{\prime}$ of $\Delta^{k} F(\sigma)=0$. Certain theorems are generalized from harmonic to polyharmonic manifolds.

\section{Introduction}

Consider a smooth $n$-dimensional Riemannian manifold $(M, g)$, the metric $g=g_{\alpha \beta} d x^{\alpha}$ $\times d x^{\beta}(\alpha, \beta=1,2, \ldots, n)$ being of any signature, i.e. properly or pseudo-Riemannian. Denote by $\nabla=d x^{x} \nabla_{\alpha}$ the Levi-Civita derivative to $g$ acting on tensor fields and by $\dot{\Delta}^{\prime}=g^{\alpha \beta} \nabla_{\alpha} \nabla_{\beta}$ the Laplace operator, where $\left(g^{\alpha \beta}\right):=\left(g_{\alpha \beta}\right)^{-1}$. Further, Synge's two-point function $\sigma=\sigma(x, y)$ is essential here; it is defined (with the help of the exponential map, cf. $\S 1$ ) for points $x, y \in M$ which are not too far from each other. For properly Riemannian manifolds $2 \sigma$ equals the square of the geodesic distance between $x$ and $y$ : $2 \sigma=r(x, y)^{2}$. Thus, $\sigma$ is the natural generalization of $r^{2} / 2$ to manifolds of arbitrary signat ure. We ask for non-constant smooth local solutions $F$ of the differential equation

$$
\Delta^{k} F(\sigma)=0
$$

i.e. for non-trivial $k$-harmonic functions which depend on the point $x \in M$ only through $\sigma=\sigma(x, y)$. Here and throughout the paper $k$ denotes a positive integer, $\Delta^{k}=\Delta \Delta \ldots \Delta$, and differential operations refer to the first argument $x$. The tern "smooth" means differentiability class. $C^{\infty}$. Distinguished signatures of $g$ are the properly Riemannian case, i.e. signature $(++\cdots+)$ or $(--\cdots-)$, and the Lorentzian case, i.e. signature $(+-\cdots-)$ or $(-+\cdots+)$. Our problem $(1)$ is a plausible 
generalization of the following two problems which have already been studied to some extent (but not yet completely solved!) and which will serve here as a guide-line:

- the theory of harmonic Riemannian manifolds, that means the problem (1) for $k=1$;

- the search for Riemannian manifolds in which a power of $\sigma$ is $k$-harmonic, that means the problem (1) for $F(\sigma)=\sigma^{l}$ or rather $F(\sigma)=|\sigma|^{l}$.

While there is a rich literature on harmonic manifolds, cf. H. S. Ruse, A. G. WALKer and T. J. Whlmore [12] and-A'. L. Besse [1] and the citations given in these books, the problem

$$
\Delta^{k}|\sigma|^{l}=0 \text {, }
$$

which had been suggested by A. GRAY to our knowledge; has been studied only recently in a series of papers by R. Caddeo, P. Matzeu, L. Vanhecke, J. FichHORN, R. SCHUMmng $[2-4,6-8,13]$.

We call a Riemannian manifold k-harmonic or polyharmonic if it admits a local solution' $F \neq$ const. of (1). Particularly,

1-harmonic $=$ harmonic, 2 -harmonic $=$ : biharmonic.

It is to be noted that, unfortunately, the term " $k$-harmonic manifold" has already been used with another meaning $[15,16]$. We think that our new terminology is a natural one and that there will not arise misunderstandings. As the first step towards the problem (1) the possible types of functions $F$ should be found out and classified. We restrict ourselves here to the class of functions $F(\sigma)$ which behave for $\sigma \rightarrow 0$ asymptotically like some power $|\sigma|^{l}$, in a sense which will be made precise in $\S 2$. The real power exponent $l$ is called the order of the function $F$ and also the order of the polyharmonic manifold $(M, g)$. We show that only the following discrete spectrum of orders $l$ is possible: for given $k$ and $n=: 2 m+2$ the number $l$ has one of the values $0,1, \ldots, k-1 ;-m, 1-m, \ldots, k-1-m$. For odd dimension $n$.half-integer orders $l$ may occur; for $n \geqq 3$ negative orders $l$ may occur. The whole order spectrum effectively occurs in any simply harmonic manifold.

We call a Riemannian manifold simply k-hairmonic or simply polyharmonic if (2) holds with some real number (the order) $l \neq 0$. Particularly, for $n \geqq 3$.

simply 1-harmonic of order $-m=$ simply harmonic.

For certain situations, i.e. assumptions on $k, l, n$ and the signature of $g$, we derive in $\$ 3$ necessary conditions on the curvature of the polyharmonic manifold. We summarize the main results in a list; the assumption on $k, l, n, g$ is followed by the conclusion on the curvature of the manifold.

1. $l=k-m-1$ or $l=k-m-2$, and $l<0$ or $n$ odd: Einstein manifold.

2. $k, l, n$ as above, $g$ Lorēntzian: constant curvature.

3. $l=k-1>0$ : constant scalar curvature.

4. $l=k-1>0, n=2$ : flat manifold.

5. $l=-1, k \leqq m$ : vanishing scalar curvature.

6. $l=-1, k=m:$ Ricci-flat manifold.

7. $l=-1, k=m, g$ Lorentzian: flat manifold.

8. $l=-1, k=m, n \geqq 6, g$ properly Riemannian: flat manifold.

We obtain these necessary conditions by means of a version of the "method of coincidence limits", which, has been developed by J. L. SyvaE [14] and B. S. DE WITT and R. W. BREнмe [5], combined with the calculus of symmetric differential forms. 
At the present state of knowledge on polyharmonic manifolds two major problems remain' open:

I. Characterize all possible types of functions $F$ in the equation (1); give a complete classification of polyharmonic manifolds.

II. Either prove that every polyharmonic manifold is harmonic or simply polyharmonic, or find a counterexample.

The second problem signals that there are good results on necessary conditions, but not so on sufficient conditions. Namely, all examples of polyharmonic manifolds which are known until now are harmonic manifolds or simply polyharmonic manifolds. Note that there exist simply polyharmonic manifolds which are not harmonic ones; in $\S 2$ we present a new class of examples for that.

\section{$\S 1$ Preliminaries}

The two-point scalar field $\sigma=\sigma(x, y)$ can be defined by more than one way. Our definition differs from $[14,5,12]$ and coincides with $[11,13]$.

Definition: Let $N(y)$ be a normal neighbourhood of $y \in M$, i.e. the exponential map with origin $y, \exp _{y}: x^{*} \mapsto x$, is a diffeomorphism from the tangential space of $y$ onto $N(y)$ or, equivalently, $N(y)$ is the domain of a normal coordinate system $x \mapsto x_{i}^{*}$ $\mapsto\left(x^{* x}\right) \equiv\left(x^{* 1}, x^{* 2}, \ldots, x^{* n}\right)$. The quantity .

$$
\sigma(x, y)=\frac{1}{2} g(y)\left(\exp _{\nu}^{-1} x, \exp _{\nu}^{-1} x\right)=\frac{1}{2} g(y)\left(x^{*}, x^{*}\right)=\frac{1}{2} g_{\alpha \beta}(y) x^{* \alpha} x^{* \beta}
$$

fór $x \in N(y)$, is called Synge's two-point function. From $\sigma$ there are derived

$$
\begin{aligned}
& e=e(x, y):=\operatorname{sign} \sigma(x, y), \quad \mu=\mu(x, y):=\frac{1}{2}(\Delta \sigma-n), \\
& \sigma_{\alpha}:=\nabla_{\beta} \sigma, \sigma^{\alpha}:=g^{\alpha \beta} \sigma_{\beta}, \quad \sigma_{\alpha \beta}:=\nabla_{\alpha} \nabla_{\beta} \sigma .
\end{aligned}
$$

The two-point scalar fields $\sigma$ and $\mu$ are ingredients of the "method of coincidence limits" $[14,5,13]$. The limit for $x \rightarrow y$, if existing, of a two-point quantity is called its coincidence limit. The equality of the coincidence limits is an equivalence relation between two-point quantities and will be denoted by $\doteq$. One-point quantities and constants may be interpreted as special two-point quantities.

We adopt the following notations and conventions.

- À symmetric differential form of degree $p$

$$
u=u_{p}=u_{\alpha_{1} \alpha_{2} \cdots \alpha_{p}} d x^{\alpha_{1}} d x^{\alpha_{2}} \ldots d x^{\alpha_{p}}
$$

is a special notation for a symmetric covariant tensor field of degree $p$ with local components $u_{\alpha_{1} \alpha}, \cdots \alpha_{p}$.

- The trace $=$ tr of a symmetric $p$-form with respect to the metric $g$ is given by

$$
\begin{aligned}
& \operatorname{tr} u_{0}=\operatorname{tr} u_{1}=0, \quad \operatorname{tr} u_{2} \pm g^{\alpha \beta} u_{\alpha \beta}, \\
& \operatorname{tr} u_{p}=g^{\alpha \beta} u_{\alpha \beta \alpha_{3} \cdots \alpha_{p}} d x^{\alpha_{1}} \ldots d x^{\alpha_{p}} \text { for } p \geqq 3 .
\end{aligned}
$$

- The trace-free part of $u_{p}$ with respect to $g$ is denoted by ${ }^{-} u_{p}$.

- The symmetric differential of $u_{p}$ is $d u_{p}: \doteq \nabla_{\beta} u_{\alpha_{1}} \therefore \alpha_{p} d x^{\alpha} d x^{\alpha_{1}} \ldots d x^{\alpha_{p}}$.

- The $p$-th power or iteration of $d$ is $d^{p}=d d \ldots d$. 
- The curvature tensor of $(M, g)$, Riem $=R_{\alpha \beta \mu \nu}\left(d x^{\alpha} \cdot \wedge d x^{\beta}\right)\left(d x^{\mu} \wedge d x^{\nu}\right)$, is defined by the Ricci identity for vector fields $v=v^{\alpha} \partial_{\alpha}$

$$
\left(\nabla_{\alpha} \nabla_{\beta}-\cdot \nabla_{\beta} \nabla_{\alpha}\right) v^{\mu}=: R_{\alpha \beta \cdot \nu}^{\mu} \cdot v^{\nu}
$$

- From Riem there are built

$$
\begin{aligned}
& \text { Ric }=R_{\alpha \beta} d x^{\alpha} d x^{\beta}=\text { Ricci tensor }:=g^{\mu \nu} R_{\alpha \mu \nu \beta} d x^{\alpha} d x^{\beta}, \\
& R=\text { scalar curvature }:=g^{\alpha \beta} R_{\alpha \beta}, \\
& (\text { Riem })^{2}:=\cdot R_{\alpha \lambda \beta \beta} R_{\mu}{ }^{2 \rho}, d x^{\alpha} d x^{\beta} d x^{\mu} d x^{\nu} ; \\
& \mid \text { Riem }\left.\right|^{2}:=R_{\alpha \beta \mu \nu} R^{\alpha \beta \mu \nu},|R i c|^{2}:=R_{\alpha \beta} R^{\alpha \beta} .
\end{aligned}
$$

The following list of "coincidence relations" is needed:

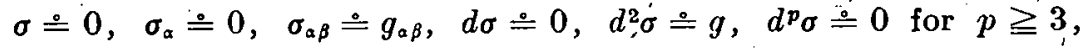

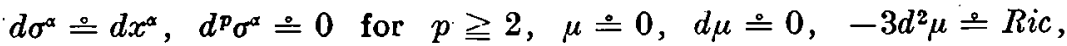

$$
\begin{aligned}
& -4 d^{3} \mu \stackrel{\circ}{=} 3 d \cdot R i c, \quad-15 d^{4} \mu \stackrel{\circ}{=} 18 d^{2} R i c+4(\dot{R i e m})^{2} \text {, } \\
& -3 \Delta \mu \stackrel{\circ}{=}, \quad-2 d \Delta \mu \stackrel{\circ}{=} d R,-15 \Delta^{2} \mu \stackrel{\circ}{=} 2 \Delta R+2\left(\mid \text { Riem }\left.\right|^{2}-\mid \text { Ric }\left.\right|^{2}\right) \text {. }
\end{aligned}
$$

For the derivation of these expressions ef. $[14,5,12,13]$.

\section{\$2 Definition and classification of polyharmonic manifolds}

Now we define the notion which gives our paper the title.

Definition 2.1: A smooth $n$-dimensional Riemannian manifold $(M, g)$ is called polyharmonic if there exist a neighbourhood $N$ of the diagonal of $M \times M$, a smooth real function $F \neq$ const. and a positive integer $k$ such that

(i) $\sigma$ is defined on $N$,

(ii) $F \circ \sigma$ is defined on $\dot{N}^{-}=\{(x, y) \in \dot{N} \downarrow \sigma(x, y) \neq 0\}$,

(iii) $\Delta^{k} F(\sigma)=0$ in $N^{-}$.

More precisely, $(M, g)$ is then called $k$-harmonic.

In the spirit of the primordial definition of a harmonic manifold $[12,1]$ one would alternatively define a polyharmonic manifold as following: there exists a function $F(\sigma, y)$ such that for each $y \in M$ and each normal neighbourhood $N(y)$ of $y$

$$
\dot{4}^{k} F(\sigma, y)=0 \text { in } N(y)^{-}=\{x \in N(y) \mid \sigma(x, y) \neq 0\} .
$$

In [12] it is made clear that for analytic manifolds and for $k=1$ the two definitions are effectively equivalent. The version choosen here is better suited to our purposes. Roughly speaking, two cases are possible for the function $F: F(\sigma)$ behaves either regular or singular for $\sigma \rightarrow 0$. J ust in order to include the singular case into the definition we demand $\Delta^{k} F(\sigma)=0$ only in $N^{-}$and not in the whole of $N$. Now we intend to refine the classification of polyharmonic manifolds.

Definition 2.2: In the situation (i), (ii) of Definition 2.1 and with a real number $l$, the function $F$ is said to be of order $l$ if $|\sigma|^{-1} F(\sigma)$ has a smooth extension $f(\sigma)$ from $N^{-}$to $N$ such that

$$
f(0) \doteq \lim _{\sigma \rightarrow 0}|\sigma|^{-1} F(\sigma) \neq .0
$$


If (iii) is satisfied too then $(M, g)$ is called a polyharmonic manifold of order $l$ or a $k$-harmonic manifold of order $l$.

This notion of an order does not cover all possible behaviours of polyharmonic functions $F(\sigma)$. The simplest counterexample is $\Delta^{k}(\log \sigma)=0$ in Euclidean space $\mathbf{R}^{2}$. We conjecture that power and logarithm functions and certain combinations of them will suffice for a complete description of the possible types of solutions $F$ of $\Delta^{k} F(\sigma)=0$. The recursive calculation $\Delta^{k}\left(|\sigma|^{l} f(\sigma)\right)=\Delta\left[\Delta^{k-1}\left(|\sigma|^{l} f(\sigma)\right)\right]$ is made precise by the following scheme.

Proposition.2.1: In the situation of Definition 2.2 the recursion with respect to $k=0,1, \ldots$

$$
\varphi_{0}^{l}=f, \varphi_{k+1}^{l}=\left[(l-k)_{\imath}(l-k+m+\mu)+(l-k) \sigma^{\alpha} \nabla_{a}+\frac{1}{2} \sigma \Delta\right] \varphi_{k}{ }^{\prime}
$$

defines smooth two-point scalar fields $\varphi_{k}{ }^{l}=\varphi_{k}{ }^{l}(x, y)$ in $N$. With these there holds $(e \Delta)^{k}\left(|\sigma|^{l} f(\sigma)\right)=2^{k}|\sigma|^{i-k} \varphi_{k}{ }^{\prime}$ in $N^{-}$.

The proof is done by means of mathematical induction with respect to $k$

Proposition 2.2: Define numbers $a_{k}{ }^{\prime}$ for $k \geqq 0$ and ${ }^{\prime} c_{k}{ }^{\prime}(p, q)$ for $k \geqq 1$ as

$$
\begin{aligned}
& a_{k}^{l}=(k !)^{2}\left(\begin{array}{l}
l \\
k
\end{array}\right)\left(\begin{array}{c}
l+m \\
k
\end{array}\right), \\
& c_{k}{ }^{\prime}(\dot{p}, q)=\sum_{r=1}^{k}[(k-r) !]^{2}\left(\begin{array}{l}
s-r \\
k-r
\end{array}\right)\left(\begin{array}{l}
t-r \\
k-r
\end{array}\right)(l-r+1) a_{r-1}^{l},
\end{aligned}
$$

where $s:=l+q, t:=l+m+p+q$. In any manifold there hold in the coincidence limit

$$
\begin{aligned}
& \varphi_{k}^{i} \stackrel{\circ}{=} a_{k}^{l} f, \quad d \varphi_{k}^{l} \stackrel{\circ}{=}, \\
& -d^{2} \varphi_{k}{ }^{l} \doteq c_{k}{ }^{l}(2,0) f^{-} d^{2} \mu \text {, } \\
& \Delta \varphi_{k}{ }^{\prime} \stackrel{\circ}{=} c_{k}{ }^{\prime}(0,1) f \Delta \mu+a_{k}{ }^{l+1} \Delta f .
\end{aligned}
$$

In an Einstein manifold there holds

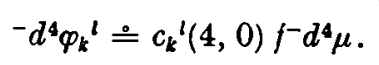

In a Ricci-flat manifold there holds

$$
\Delta^{2} \varphi_{k}^{l} \stackrel{\circ}{=} c_{k}^{l}(0,2) f \Delta^{2} \mu+a_{k}^{l+2} \Delta^{2} f
$$

Proof: Successive differentiation of (2.1) gives for any manifold

$$
\begin{aligned}
& \varphi_{k+1}^{l} \doteq(l-k)(l-\ddot{k}+m) \varphi_{k}^{\prime}, d \varphi_{k+1}^{l} \stackrel{\circ}{=}(l-k)(l-k+m+1) d \varphi_{k}^{\prime}, \\
& -d^{2} \varphi_{k+1}^{l} \doteq(l-k)(l-k+m+2)-d^{2} \varphi_{k}{ }^{l}+(l-k)-d^{2} \mu \varphi_{k}{ }^{l} \text {, } \\
& \Delta \varphi_{k+1}^{l} \stackrel{\circ}{=}(l-\dot{k}+1)(l-k+m+1) \Delta \varphi_{k}{ }^{\prime}+(l-k) \Delta \mu \varphi_{k}^{\prime} \text {, }
\end{aligned}
$$

and for an Einstein manifold

$$
-d^{4} \varphi_{k+1}^{l} \stackrel{\circ}{=}(l-k)(l-k+m+4)-d^{4} \varphi_{k}{ }^{l}+(l-k)-d^{4} \mu \varphi_{k}{ }^{l},
$$

and for a Ricci-flat manifold

$$
\Delta^{2} \varphi_{k+1}^{l} \stackrel{\circ}{=}(l-k+2)(l-k+m+2) \Delta^{2} \varphi_{k}^{l}+(l-k) \Delta^{2} \mu \varphi_{k}^{l}
$$


These recursion equation systems with respect to $k=1,2, \ldots$ are completed by the initial values

$$
\cdot \varphi_{0}{ }^{\circ} \doteq f, \quad d \varphi_{0}{ }^{l} \doteq 0, \quad-d^{2} \varphi_{0}{ }^{i} \doteq 0, \quad \Delta \varphi_{0}{ }^{l} \doteq \Delta f, \quad-d^{4} \varphi_{0}{ }^{l} \doteq 0, \quad \Delta^{2} \varphi_{0}{ }^{l} \doteq \Delta^{2} f .
$$

Considering that the numbers $a_{k}{ }^{l}, c_{k}{ }^{l}=c_{k}{ }^{l}(p, q)$ are the unique solutions of the recursions

$$
\begin{aligned}
& a_{0}{ }^{l}=1, \quad a_{k+1}^{l}=(l-k)(l-k+m) a_{k}{ }^{\prime}, \\
& c_{0}{ }^{l}=0, \quad c_{1}{ }^{l}=l ; \quad c_{k+1}^{l}=(s-k)(t-k) c_{k}{ }^{l}+(l-k) a_{k}{ }^{l},
\end{aligned}
$$

we verify that $(2.2)-(2.6)$ are indeed the solutions of the recursion equation systems under question

Note that the numbers $c_{k}{ }^{l}(p, q)$ are the same ones as in [13]. Note further that

$$
\begin{aligned}
& 2 c_{k}^{l}(0,1)=k(2 l-k+1) a_{k-1}^{l}, \\
& 2 c_{k}{ }^{l}(2,0)=k !(k-2) ! k(2 l+2 m+3-k)\left(\begin{array}{l}
l \\
k
\end{array}\right)\left(\begin{array}{l}
l+m \\
k-2
\end{array}\right) \text { for } k \geqq 2 .
\end{aligned}
$$

Our method of derivation of necessary conditions on polyharmonic manifolds will be the following. The two-point condition $\Delta^{k}\left(|\sigma|^{l} f(\sigma)\right)=0$ in $N^{-}$is continued to the ' equivalent two-point condition $\varphi_{k}{ }^{\prime}=0$ in $N$. The latter is evaluated through a set of necessary one-point conditions, namely' $d^{p} \varphi_{k}{ }^{l} \doteq 0$ for $p=0,2,4$ and $A^{q} \varphi_{k} \stackrel{\circ}{=} 0$ for $q=1,2$. Our first result obtained this way is a restriction to the values of the order $l$.

Theorem: If a k-harmonic manifold of dimension $n=2 m+2$ possesses an order $l$ then $l$ is an integer with $0 \leqq l \leqq k-1$ or $l+m$ is an integer. with. $0 \leqq l+m$ $\leqq k-1$.

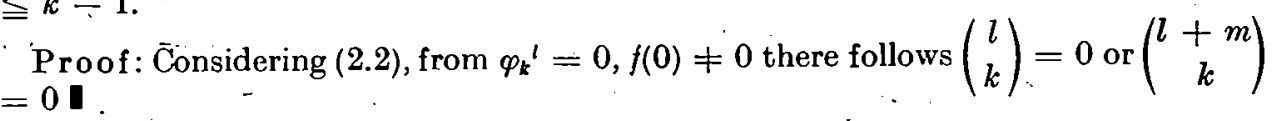

The question arises whether all orders compatible with the theorem effectively occur. It is answered to the positive by the following examples.

Example 2.1 [13]: Every simply harmonic manifold fulfills

$$
\begin{aligned}
& \Delta^{k} \sigma^{k-1}=0 \text { for } k=2,3, \ldots, \\
& \Delta^{k} \sigma^{k-m-1}=0 \text { for } k=1,2, \ldots
\end{aligned}
$$

Example 2.2: Every generalized plane-wave manifold, which is characterized by a metric of the form

$$
g=2 d x^{1} d x^{2}+g_{i j}\left(x^{1}\right) d x^{i} d x^{j} \quad(i, j=3,4, \ldots, n),
$$

fulfils (2.7).

Proof: A procedure analogous to the proof of Proposition 2.1 shows that there exist smooth functions $f_{k}{ }^{l}\left(x^{1}\right)$ of the first coordinate only such that

$$
(e \Delta)^{k}\left(|\sigma|^{l}\right)=f_{k}^{l}\left(x^{1}\right)|\sigma|^{l-k} \text {. }
$$

The special properties of the metric (2.9) are used in the induction step. Particularly, $\mu$ is a function of $x^{1}$ only and every smooth function of $x^{1}$ only is a harmonic function, cf. P. GÜNTher [9]. As a consequence of (2.10), $A^{k-1} \sigma^{k-1}$ depends on $x^{1}$ only and is therefore a harmonic function, i.e. $\Delta^{k}\left(\sigma^{k-1}\right) \equiv \Delta\left(\Delta^{k-1} \sigma^{k-1}\right)=0 \mathrm{~d}$, 
From (2.7) there follows for $l=0,1, \ldots, k-1$ and for any polynomial (with constant coefficients) $f$ of degree $\leqq k-1-l$ that $\Delta^{k}\left(\sigma^{l} f(\sigma)\right)=0$. Analogously, the last follows from (2.8) for $l+m=0,1, \ldots, k-1$ and $f$ as above. While Example 2.1 has been quoted in [13], Example 2.2 has been given in [13] only for $k=2$. The generalized plane-wave manifolds may have any dimension $n \geqq 3$ and any signature different from the properly Riemannian one. They are non-harmonic simply polyhärmonic manifolds. Another class of non-harmonic simply biharmonic manifolds of order $l=1$ has been given by R. CADDEO and P. MATZEU [3]: these are products of three-dimensional manifolds of constant curvature and had been suggestéd by A. Gray.

\section{$\$ 3$ Necessary conditions for polyharmonic manifolds}

Every harmonic manifold is an Einstèin manifold [12; 1]. This well-known fact has a generalization to polyharmonic manifolds.

Theorem 3.1: Every $k$-harmonic manifold of negative or half-integer order $l=k-m$ - 1 or $l=k-m-2$ is an Einstein manifold.

Proof: For $k=1$ the mentioned theorem on harmonic manifolds enuérges. For $k \geqq 2$ exactly under the assumed conditions on $l$ the factor $c_{k}{ }^{\prime}(2,0)$ in $-3-d^{2} \varphi_{k}{ }^{l}$

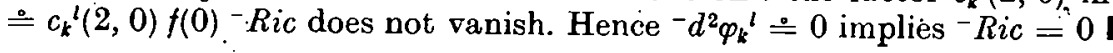

Example 3:1: Every biharmonic manifold of negative or half-integer order is an Einstein manifold.

As A. Lichinerowicz and A. G. WALkER [10] found out, every harmonic mianifold with a Lorentzian metric is of constant curvature. This important theorem too has a generalization to polyharmonic manifolds.

Theorem 3.2: Every Lorentzian k-harmonic manifold of negative or half-integer order $l=k-m-1$ or $l=k-m-2$ has constant curvature.

Proof: Theorem 3.1 tells us $-R i c=0, d R i c_{1}=0,-15 d^{4} \mu \doteq 4(\text { Riem })^{2}$. Thus the necessary condition based on $(2.5)$ reads $0 \stackrel{\circ}{=}-15^{-} \cdot d^{4} \varphi_{k}^{l} \stackrel{\circ}{=} 4 c_{k}^{l}(4,0) f(0)^{-}(\text {Riem })^{2}$. For $k=1$ the mentioned theorem on Lorentzian harmonic manifolds emerges. For $k=2,3$ a direct calculation shows that each of the numbers $c_{2}{ }^{1-m}(4,0), c_{2}{ }^{-m}(4,0)$, $c_{3}{ }^{2-m}(4,0), c_{3}{ }^{1-m}(4,0)$ does not vanish. For $k \geqq 4$ we have the situation of [13: Prop. $2.6 /(\mathrm{iv})]$; hence $c_{k}{ }^{\prime}(4,0) \neq 0$. Summarized, - Ric $=0,-(\text { Riem })^{2}=0$ holds for every $k$. In the Lorentzian case this implies constant curvature $[10,12,13]$

Example 3.2: Every Lorentzian biharmonic manifold of negative or half-integer order has constant curvature.

Clearly, the Einstein condition in Theorem 3.1 implies $R=$ const. There is also another situation with $R=$ const.

Theorem 3.3: Every $k$-harmonic manifold of order $l=k-1>0$ has constant scalar curvature.

Proof: The factor $c_{k}^{k-1}(0,1)$ in $c_{k}^{k-1}(0,1) f(0) R=3 a_{k}^{k}(\Delta f)(0)-3 \Delta \varphi_{k}^{k-1}$ does not vanish. Hence $\Delta \varphi_{k}^{k-1} \doteq 0$ implies $R=$ const

Example 3.3: Every two-dimensional $k$-harmonic manifold of order $l=k-1>0$ is flat.

Proof: Theorem 3.3 tells is $R=$ const. A calculation for a two-dimensional manifold of non-vanishing constant curvature, following e.g. [2], shows: either contains 
the solution $F(\sigma)$ of $\Delta^{k} F(\sigma)=0$ logarithmic terms or $F(\sigma)=$ const. Both these cases - are not allowed here; hence $R=0$ I

'Theorem 3.4: A k-harmonic manifold of order $l=-1$ with $k \leqq m$ has vanishing scalar curvature and even dimension $n \geqq 4$.

Proof: The order $l=-1$ can occur only for even $n \geqq 4$. For $k \leqq m$ the factor $c_{k}{ }^{(-1)}(0,1)$ in $c_{k}{ }^{(-1)}(0,1) f(0) R \doteq 3 a_{k}^{0}(\Delta f)(0)-3 \Delta \varphi_{k}{ }^{(-1)}$ does not vanish, while $a_{k}{ }^{0}$ $=0$. Hence $\Delta \varphi_{k}(-1) \stackrel{\circ}{=} 0$ implies $R=0$

Theorem 3.5: An m-harmonic manifold of order $l=-1$ is Ricci-flat. A Lorentzian $m$-harmonic manifold of order $l=-1$ is flat.

Proof: Both the Theorems 3.1 and 3.4 apply to the present situation $k=m$, $l=-1$. Analogously, both the Theorems 3.2 and 3.4 apply to the situation $k=m$, $l=-1, g$ Lorentzian

Theorem 3.6: A properly Riemannian m-harmonic manifold of order $l=-1$ and even dimension $n \geqq 6$ is flat.

Proof. 'Theorem 3.5 tells us $R i c=0$. Thus the necessary condition based on (2.6) reads

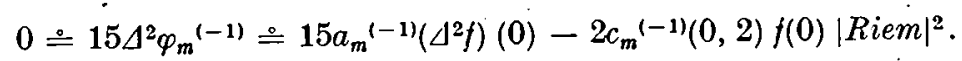

Here the factor $c_{m}{ }^{(-1)}(0,2)$ does not vanish for $k=m \geqq 2$ according to [13:Prop. 2.6], while $a_{m}{ }^{(-1)}=0$. Hence $\mid$ Riem $\left.\right|^{2}=0$. In the properly Riemannian case this implies flatness :

Example 3.4: A six-dimensional biharmonic manifold of order $l=-1$ is Ricciflat. A Lorentzian or properly Riemannian six-dimensional biharmonic manifold of order $l=-1$ is flat.

Inspection of the formulas (2.4), (2.6) together with $\Delta f \stackrel{\circ}{=} f^{\prime}(0), \Delta^{2} f \stackrel{\circ}{\doteqdot}(n+2) f^{\prime \prime}(0)$ $+2 \Delta \mu f^{\prime}(0)$ shows that more theorems on polybarmonic manifolds could be produced by making the assumption $f^{\prime}(0)=0$ or $f^{\prime \prime}(0)=0$. We omit here such considerations because they are less interesting at the moment. We think that neither the "method of coincidence limits" is exhausted by the present paper nor is it the only possible approach to polyharmonic manifolds.

\section{REFERENCES}

[1] B́Esse, A. L.: Manifolds all of whose Geodesics are Closed. Berlin : Springer-Verlag 1978.

[2] CADDEO, R.: Riemannian manifolds on which the distance function is biharmonic. Rend. Sem. Mat. Univ. Politecn. Torino 40 (1982), 93-101.

[3] Cadveo, R., and P. Matzeu : Riemannian manifolds with $\Delta^{2} r^{k}=0$. Preprint, Universitá di Cagliari 1983.

[4] CADDEO, R., and L. VANHECKE: Does $\Delta^{2} d^{2-\dot{n}}=0$ on a Riemannian manifold imply flátness? Period. Math. Hung. 17 (1986), 109-118.

[5] De WItT, B. S., and R. W. Bremse: Radiation Damping in a Gravitational Field. Ann. Physics 9 (1960), 220-259.

[6] Eıcheors, J.: Lokale Geometrie des Radius in Riemannschen Mannigfaltigkeiten I. Beitr. z. Alg. u. Geom. 18 (1984), 41-48.

[7] Eichнов , J.: Lokale Geometrie des Radius in Riemannschen Mannigfaltigkeiten II. Beitr. z. Alg. u. Geom. 18 (1984), 177-189.

[8] Eichmorn, J., und R. Schimirna: Riemannsche Mannigfaltigkeiten, bei denen eine Potenz des Radius $k$-harmonisch ist. Potsdamer Forschungen, Naturw. R. 43 (1984), 74-80. 
[9] Güsther, P.: Ein Beispiel einer nichttrivialen Huygensschen Difféèntialgleichung mit vier unabhängigen Veränderlichen. Arch. Rational Mech. Anal. 18 (1965), 103-106.

[10] Lichnerowicz, A., et A. G. Walker: Sur les espaces riemanniens harmoniques de type hyperbolique normal. Compt. Rend. Acad. Sc. Paris 221 (1945), 394-396.

[11] Peleska; J.: Zur Charakterisierung isometrischer und konformer Abbildungen zwischen pseudo-Riemannschen Mannigfaltigkeiten. Dissertation. Universität Hamburg 1982.

[12] Ruse, H. S., Walker, A. G., and T. J. Wrlmore: Harmonic Spaces. Roma: Edizioni Cremonese 1961.

[13] Scmmma, R.: Riemannian manifolds for which a Power of the Radius is $k$-harmonic. Z. Anal. Anw. 4 (1985), 235-249. [14] Synge, J. L.: Relativity. The general theory. Amsterdam: North-Holland Publ. Comp.
1960.

[15] WrLlmore, T. J.: 2-Point Invariant Functions and $k$-Harmonic Manifolds. Rev. Roum. Math. Pur. Appl. 13 (1968), $1051-1057$.

[16] WILlmore, T. J., and J. El HaDI: $k$-harmonic symmetric manifolds. Rev. Roum. Math. Pur. Appl. 15 (1970), 1573-1577.

Manuskriptéingang: 23.05. 1985

VERFASSER:

Doz. Dr. sc. Rajiver Schmmlvg

Sektion Mathematik der Ernst-Moritz-Arndt-Universität

Friedrich-Ludwig-Jahn-Str. 15a, DDR-2200 Greifswald

Dr. JAN KowolIK

Instytut Matematyki, Wyzsza Szkola Pedagogiczna

ul. Oleska 48, P-45.052 Opole 